Center for Astrophysics

Preprint Series No. 3959

(octobe 7,1934)

\title{
INTERPRETATION OF GRAVITATIONAL MICROLENSING BY BINARY SYSTEMS
}

S. Mao and R. DiStefano

Harvard-Smithsonian Center for Astrophysics

and
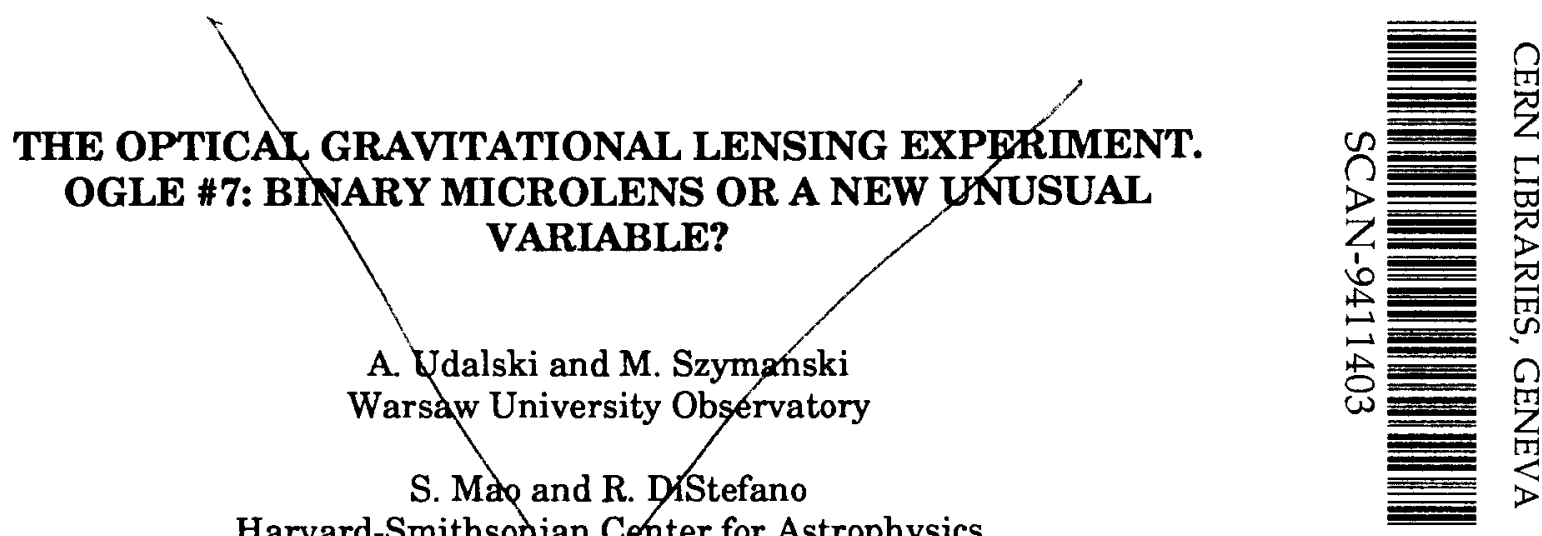

Harvard-Smithsonian Center for Astrophysics

J. Kaluznyand M. Kubiak

Warsaw University Observatory

M. Matep

509448

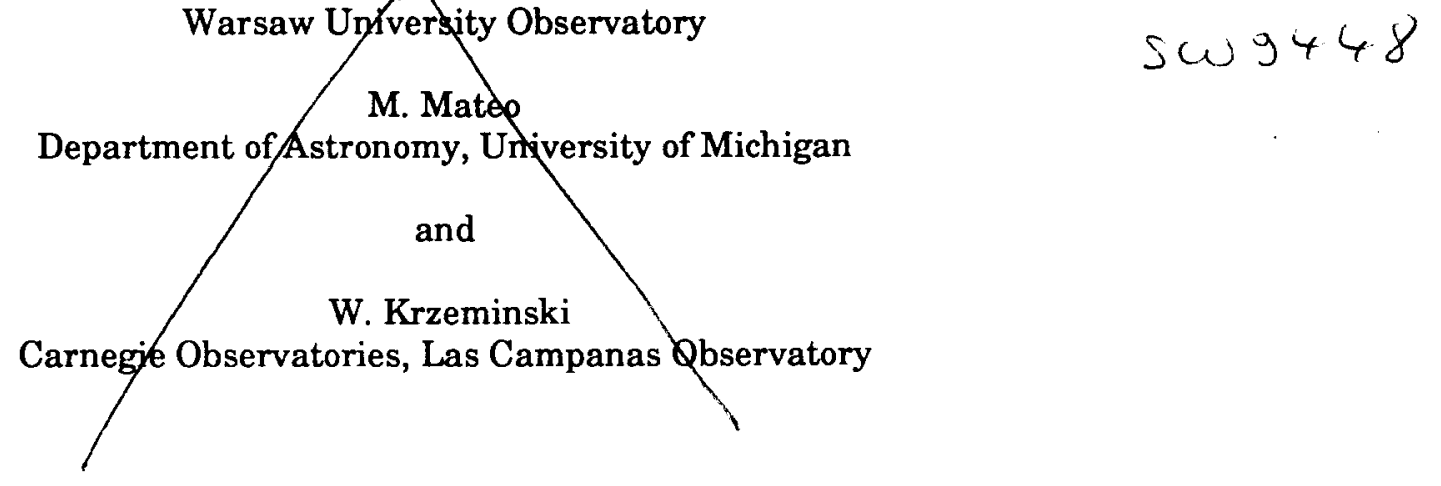

$$
\begin{aligned}
& \text { To appear in } \\
& \text { The nstrophysical Sownal (Letters) } \\
& \text { December 1, } 1934
\end{aligned}
$$




\title{
Interpretation of Gravitational Microlensing by Binary Systems
}

\author{
S. Mao and R. DiStefano \\ MS 51, Center for Astrophysics, 60 Garden Street, Cambridge, MA 02138
}

\begin{abstract}
The fraction of massive compact halo objects (MACHOs) that may be binary systems is unknown, but there is no a priori reason to assume that it is small. If stellar systems can provide useful guidelines, then the fraction may be close to one-half. It is therefore possible that some of the microlensing events, including both those due to stellar systems and those due to MACHOs, may exhibit the complex light curves associated with binarity. In this work we propose a method to search for evidence of binarity in the microlensing light curves and demonstrate that our approach can be used to extract binary parameters directly from the light curves. The backdrop of our method is the establishment of a library of binary events where the most important features (including the positions, amplitudes, and measures of asymmetry) are stored. This library allows us to identify a relatively small number of candidate events that mimic the main features of any given light curve. These candidate events provide the input for a $\chi^{2}$ minimization routine which identifies the parameters associated with the best fit. We find that for weak and moderate binary events, the solutions can be degenerate while for strong events, although the process of deriving the best fit curve generally requires more work, the resulting solution tends to be unique.

While a densely sampled light curve and good photometry can better constrain the binary parameters, we find that samplings that are well within the reach of the current generation of observational programs should be sufficient to shed light on the issue of what fraction of lensing objects are in binaries, and on some of the properties of the binaries themselves.
\end{abstract}

Subject headings: the Galaxy: halo - gravitational lensing - binaries: general

\section{Introduction}

Searches for gravitational microlensing events through long-term monitoring of millions of stars in the Large Magellanic Cloud (LMC) and the Galactic bulge are beginning to discover exciting candidates (Alcock et al. 1993; Aubourg et al. 1993; Udalski et al. 
1993, 1994a). The primary goal of these searches is to probe for the existence of massive compact halo objects (MACHOs) via their gravitational microlensing effect on background stars (Paczyński 1986, 1991; Griest 1991).

The majority of disk stars are in binaries (see, e.g., Abt 1983; Mazeh et al. 1992). Mao \& Paczyński (1991) estimated roughly $10 \%$ of the microlensing light curves of the sources in the Galactic bulge should show strong binary signatures. There is no similar estimate for MACHOs in our Galactic halo, as we have no information about the binarity of MACHOs. It is therefore important to address the question of how to extract information about disk and MACHO binarities from the observed light curves. Indeed, our ability to identify binary lens induced light curves is crucial: since such light curves can have characteristics that are very different from those due to a single lens, a large number of events would fail to pass the standard requirement of symmetry associated with point-mass lenses. The features of binary lens events have been studied by many authors (e.g. Mao \& Paczyński 1991 and references therein). The associated light curves can exhibit significant deviations from symmetry and can have multiple peaks, and even single peaks can be exaggerated relative to the peaks seen for isolated lenses. Features that binary and single lenses have in common are that the variations in the light curves should be achromatic. We note, however, that achromaticity may be violated occasionally for binary lenses, for binary sources, when the source cannot be modeled as a point object and also for single lenses when the lenses are also shining. Although a small fraction of binaries may generate two events as the projection of a single source transits the lens plane, long-term repetition of the variation is highly unlikely.

The successes of different groups have demonstrated that both the hardware and software of microlensing searches are working; it is therefore a matter of time, and may already have happened (Udalski et al. 1994b), that some peculiar events are observed by one of the systematic searches for gravitational microlensing. The interpretation of these events is important, as they may provide valuable information about the true spatial density of MACHOs, about the binarity of MACHOs, and about planetary systems (Mao \& Paczyński 1991; Gould \& Loeb 1992; Bolatto \& Falco 1994). Several questions naturally arise: how can we identify that a light curve is due to a binary lens? can we determine the relevant binary parameters and the uncertainties in those parameters? In this paper, we demonstrate that a method based on a systematic study of light curves associated with binaries can help to answer the above questions. The outline of the paper is as follows: in $\S 2$, we review the basic notation; in $\S 3$ we describe our approach and give examples of its application, in $\S 4$, we briefly discuss our results. 


\section{Basic Notation}

A point mass lens is characterized simply by its mass, $M$. The light curve induced by a point mass is completely defined by the distance of closest approach, $b$, between the lens and the projection of the source onto the lens plane and the time of closest approach, $t_{b}$. In contrast, a binary is characterized by its total mass, $M$; the mass ratio of the individual masses, $q=m_{2} / m_{1}$; their separation, $a$, as projected onto the lens plane (defined in terms of the center of mass of the binary). The source trajectory is determined by the closest approach to the center of mass, $b$; and $\theta$, the angle between the axis of the binary and the trajectory. The geometry of binary lensing is illustrated in Fig. 1.

Throughout the paper, the length scales are projected onto the lens plane and are normalized to the Einstein radius on the lens plane,

$$
R_{E}=\left(\frac{4 G M D_{d} D_{d s}}{D_{s} c^{2}}\right)^{1 / 2}=4.3 \times 10^{13} \mathrm{~cm}\left(\frac{M}{0.1 M_{\odot}}\right)^{1 / 2}\left(\frac{D_{s}}{10 \mathrm{kpc}}\right)^{1 / 2}[x(1-x)]^{1 / 2}
$$

where $D_{d}, D_{s}$ and $D_{d s}$ are respectively the distance to the lens, the distance to the source, and the distance between the lens and the source, and $x=D_{d} / D_{s}$ (see Blandford \& Narayan 1992 for a review). The duration of a lensing event, $t_{E}$, is usually defined as the time taken for the source to travel one Einstein radius across the lens plane:

$$
t_{E}=\frac{R_{E}}{v_{t}}=25 \text { days }\left(\frac{M}{0.1 M_{\odot}}\right)^{1 / 2}\left(\frac{D_{s}}{10 \mathrm{kpc}}\right)^{1 / 2}[x(1-x)]^{1 / 2}\left(\frac{v_{t}}{200 \mathrm{~km} \mathrm{~s}^{-1}}\right)^{-1}
$$

where $v_{t}$ is the transverse velocity projected on the lens plane between the lens, the source and the observer. From Eqs. (1) and (2), we see that for lensing in the LMC or the Galactic halo, the Einstein radius is a few $\mathrm{AU}$, and the duration is roughly 1 month for a $0.1 M_{\odot}$ object.

For an isolated lens, the measured characteristics of the light curve allow one to fit values for $b, t_{b}, t_{E}$, and the unlensed magnitude of the star, $m_{0}$. Knowledge of these values, together with assumptions about the likely values of relative distances and velocities, allow one to place limits on $M$. For a binary lens, in addition to needing to find values of $b, t_{b}, t_{E}$ and $m_{0}$, we also need to fit for $a, q$, and $\theta$. Fortunately, binary light curves have more features than those associated with isolated point masses; it is the additional features that make the work described here possible.

In order to refine our method, we needed to be able to test it on a large number of binary lensing events that were sampled in a way that mimics the observational capabilities of the present generation of observational programs. We generated the parameters for the 
binary and the source trajectory of each test event randomly. The associated theoretical light curves were sampled evenly, with a sampling frequency of $\Delta l_{0}$. The errors of photometry are assumed to be Gaussian and follow Poisson statistics,

$$
\sigma=\sigma_{0} 10^{0.2\left(m-m_{0}\right)}
$$

where $m_{0}$ is the unlensed magnitude of the star and $\sigma_{0}$ is the measurement error at magnitude $m_{0}$. This expression simulates how the measurement error in magnitude changes when the star becomes brighter as a result of lensing. Observed light curves have typical errors between 0.01-0.1 magnitude depending on the brightness of the star; typical durations are 10-40 days, so a daily observation would roughly correspond to $\Delta l_{0}=0.025-0.1$ in units of the Einstein radius. We will use the average values of the measurement error and sampling frequency: $\Delta l_{0}=0.05, \sigma_{0}=0.05$. We adopt $t_{E}=1$ or -1 (the signs indicating two possible directions), $m_{0}=0$ when generating the light curves but allow them to vary in fitting.

\section{Overview of the Method}

In our model, there are six parameters that specify the binary geometry and the trajectory of the source and one additional parameter to specify the unlensed magnitude of the star. Given a light curve, the standard approach to finding these parameters is to minimize $\chi^{2}$, the deviation of the fitted light curve from the "observed" one:

$$
\chi^{2}=\sum_{i=1}^{N_{\mathrm{obs}}}\left(\frac{m_{i, f}-m_{i, o b s}}{\sigma_{i}}\right)^{2},
$$

where $N_{\text {obs }}$ is the number of data points, $m_{i, f}$ and $m_{i, o b s}$ are the fitted and "observed" magnitudes, and $\sigma_{i}$ is the measurement error. A more rigorous approach is to use the flux weighted $\chi^{2}$, but for the small errors we discuss in this paper, eq. (4) gives nearly identical results. The theoretical light curve can be computed using the complex notation developed. by Witt (1990). With this method, the lens equation can be simplified into a fifth order polynomial, which then can be solved numerically using the Laguerre's method (see Press et al. 1992). We use the Levenberg-Marquardt method in Press et al. (1992) to minimize $\chi^{2}$.

As a result of the vast diversity of binary light curves, the function $\chi^{2}$ has many local extrema. Not surprisingly, the minimization routine sometimes converges to a local minimum close to the initial guesses of the parameters instead of the desired global minimum. It is therefore necessary to supplement the standard procedure. We have chosen to do so by using a comprehensive study of binary light curves (described below). This enables us derive a small set of candidate events for each observed (or generated) event. These candidates then serve as the initial guesses to the $\chi^{2}$ minimization routine. 


\subsection{Features of Binary Light Curves}

We have systematically sampled the parameter space of binary light curves, by varying the binary separation, $a$, from $0.01 R_{E}$ to $3.5 R_{E}$, the mass ratio, $q$, from $10^{-4}$ to $10^{4}$, the distance of closest approach across the same range of values as $a$, and the angle of incidence, $\theta$ from $0^{\circ}$ to $180^{\circ}$. The salient features of each light curve were computed and recorded, to provide us with a library of events, against which any observation can be compared. The features that can be related to the physical parameters include the locations and magnitudes of maxima and minima, and various measures of asymmetry.

In this work we focus on multiple-peaked events. We have found that, for these events, the positions and amplitudes of the maxima and minima correlate well enough to the physical parameters, that a search of our library based on just those features identifies good candidate events in the parameter space. These candidates are then used as the initial parameters for the $\chi^{2}$ fitting procedure. The presence of multiple peaks is generally a sign that the source track passes directly through or comes close to the fold or cusp caustics. It is convenient to consider two subsets of multiple-peaked events, which we have chosen to call "strong binary events" and "weak binary events." In the following, we give three examples to illustrate our fitting procedure.

\subsection{Strong Binary Events}

In Fig. 2, we show one example of a strong binary event. To fit the light curve, we search through our library of events to identify candidates that have at minimum the number of observed extrema, with roughly the same positions and amplitudes as those observed for this particular event. The procedure for conducting such a search must be sensitive to the fact that we are comparing the well-sampled members of our library of events to an observed event which may not display all of the features that it would have displayed, had it been sampled more frequently. In this case, we identified only those events for which the following conditions apply:

1. there are at least three peaks,

2. the magnitude of the first peak lies between -0.3 and -0.65 ,

3. the magnitude of the final peak is brighter than -1.2 ,

4. the time elapsed between the first peak and the last peak is at least $25 \%$, but no more than $70 \%$, of the duration of the entire event (estimated as one half of the time 
interval between the first and last points above $0.3 \mathrm{mag}$, and allowing roughly a factor of 2 uncertainty), and

5. there is at least one peak between the first and final peaks which is brighter than -1.8 .

Just 17 candidate events emerge from this search of 37,623 . Of these, 13 of the events from the library have three peaks, and four have four peaks. When each of these are used as initial conditions to the $\chi^{2}$ fitting routine, the best-fit curve shown in Fig. 2 emerges as the global minimum, and therefore, the only viable possibility. The parameters are:

$$
q=2.75, a=1.97, b=0.52, \theta=22.6^{\circ}, t_{E}=1.02, t_{b}=-1.04, m_{0}=0.0 .
$$

and the associated value of $\chi^{2}$ is 161 for 153 degrees of freedom. These parameters are well fixed and should be compared with the "true" solution:

$$
q=2.49, a=1.97, b=0.49, \theta=21.7^{\circ}, t_{E}=1.0, t_{b}=-1.0, m_{0}=0.0 \text {. }
$$

In Fig. 2, the corresponding caustics and trajectory are shown. Notice the correspondence of the features in the light curve and the caustic structures.

\subsection{Weak Binary events}

In Fig. 3, we show an example of a light curve that is more slightly perturbed than the one considered above. A single lens model produces just one peak and therefore cannot well reproduce the observed light curve. This is reflected in the large value of $\chi^{2}=300$ for the best point-mass model (not shown) with 156 degrees of freedom. We therefore proceed with our method for identifying binarity.

In this case, because the amplification is relatively small, it is difficult to know whether there may have been small peaks that were missed, either before or after the two peaks near the center. In this case, the search of the library looked for all events for which the following is true:

1. there are two or more peaks,

2. two of the peaks have magnitudes brighter than -0.4 ,

3. these two peaks are separated by a time which is between $5 \%$ and $20 \%$ of the duration of the event, and

4. there is a minimum, located roughly halfway between the two peaks, with a magnitude between -0.3 and -0.45 . 
Fourteen candidate events were identified by the search, and each of these was used as input for the $\chi^{2}$ fit. The results were three sets of parameters which gave satisfactory fits. Two of these are shown in Fig. 3. The parameters are, respectively,

1. $q=0.037, a=0.8, b=0.7, \theta=85^{\circ}, t_{E}=0.83, t_{b}=-0.40, m_{0}=-0.02$; and

2. $q=0.38, a=1.28, b=0.80, \theta=131.7^{\circ}, t_{E}=-0.96, t_{b}=-0.66, m_{0}=-0.01$.

The $\chi^{2}$ for these two models are respectively 182 , and 156 , with 153 degrees of freedom, and therefore are both within $2 \sigma$ confidence limit. The results can be compared with the true solution,

$$
q=0.38, a=1.26, b=0.77, \theta=136.7^{\circ}, t_{E}=-1.0, t_{b}=-0.65, m_{0}=0.0 .
$$

The caustic structures and source trajectories for these two binary models are dramatically different. However, both models are able to reproduce the light curve, because both trajectories pass through two cusps producing the two gradual peaks (see Fig. 3). The biggest difference between these two models is the mass ratio. They differ by a factor of 10 . Interestingly, if the primary lens has a mass of $0.1 M_{\odot}$, the mass of the secondary in the first binary model will have a mass 4 times that of Jupiter, in the range of planetary systems.

\subsection{An Example From the Observations}

In this section we will apply our algorithm to the real data obtained by the Optical Gravitational Lensing Experiment (OGLE, Udalski et al. 1993, 1994a).

In Fig. 4, the lensed part of the light curve for the OGLE \#6 event is shown. The sampling in the light curve is uneven and rather sparse. The accuracy of photometry is $\approx 0.05$ mag. at minimum light. The best point lens model (shown as a dashed line) gives $b=0.145, t_{E}=8.4 \mathrm{~d}, t_{b}=818.9 \mathrm{~d}, m_{0}=18.1$, with a $\chi^{2}=198$ for 91 degrees of freedom. Owing to non-Gaussian errors in the photometry, the significance of this $\chi^{2}$ is difficult for us to evaluate. However, there seems to be some indication of another peak around 835d. Unfortunately this impression is associated with a small number of points, especially by the data point at $840 \mathrm{~d}$. Having this caveat in mind, we can nevertheless search our library for events with one or more peaks and roughly match the peak positions and amplitudes. Eighteen candidates turned up in our search. These candidates are then used as input of our minimization routine. The best solution is shown in Fig. 4 together with its lensing geometry. The parameters are

$$
q=0.26, a=2.38, b=0.16, \theta=6.2^{\circ}, t_{E}=9.0 \mathrm{~d}, t_{b}=822.7 \mathrm{~d}, m_{0}=18.07,
$$


with a $\chi^{2}=146$ for 88 degrees of freedom. The improvement is more impressive if we examine only the lensed portion of the light curve (between $800 \mathrm{~d}$ and $850 \mathrm{~d}$ ): $\chi^{2}$ shows a dramatic improvement from 75 to 26 for 31 data points. This illustrates that the non-varying wings of a light curve tend to damp out the deviation signal from a single point mass model.

\section{Discussion}

The analysis presented here represents the first systematic attempt to extract binary parameters from light curves. To isolate the main effects, we have ignored the finite source size and the orbital motions of the binary, while in reality they may provide small perturbations.

Our pragmatic fitting procedure first attempts to find the best initial parameters by matching the global features in the observed light curve. These conditions are then used to search for the $\chi^{2}$ global minimum. This two-step procedure proves successful, although some intuitive understanding of caustics and trajectories is still useful. For the strong events studied by Mao \& Paczyński (1991), the physical parameters are usually unique owing to the distinctive features in the light curve. For weaker events the solutions are possibly degenerate, and can only be broken by better observational sampling and photometry. For the example in Fig. 3, the maximum magnitude difference between the two binary models is roughly 0.05 magnitude. Sampling three times more frequently or improving the photometry by a factor of 2 will unambiguously distinguish these two models. In addition, there is a question of whether all the acceptable degenerate solutions are achieved with equal probability in the parameter space. Such evaluation requires a detailed knowledge of the binary population and the individual experiments.

Due to the enormous diversity in the light curves, some types of variability may well be fitted by binary lenses. However, the problem is not much worse than that for the identification of a lens candidate by a single mass. One requires the light curves to be achromatic in multiband observations and the variability not repeated with time. These two requirements are essential in the identification of binary events in order to avoid false positives, although we caution that occasional achromaticity may occur when the source is not small compared with the Einstein radius or/and when the source comes close to the caustics. These violations generally occur when the magnifications are high. When the observation is only available in one band, although the characteristic lensing features may lend support to the binary lensing interpretation, some ambiguity may remain.

The caustic crossing in the strong events on average last for only $\approx 0.06 t_{E}$ (Mao \& Paczyński 1991), therefore a binary event can be easily missed if sampled irregularly or not 
frequently enough. Fortunately this required frequent sampling can now be achieved. For example, the OGLE group has successfully implemented the "early warning system" (Udalski et al. 1994c), where a lens candidate can be identified when it is still in the rising branch and can be monitored frequently by observers all over the world. A well-sampled binary light curve allows one to determine all the parameters accurately. Furthermore, the mass of the lens can be deduced if the orbit of the binary can be determined spectroscopically.

Our study is the first step in understanding the essentials in interpreting the binary lensing events. This method has been successfully applied to OGLE \#7, a promising lensing candidate discovered in the gravitational lens search in the OGLE data. The results are reported elsewhere (Udalski et al. 1994b). However, many quantitative questions about binary lensing remain to be answered, including the relative frequency of asymmetric singlepeak events to the multiple peak events, and their detectability in real observations. These issues will be addressed in a forthcoming paper (Di Stefano \& Mao 1994). It is also our goal to develop a software system that automates this procedure for use by the observational groups.

We thank Bohdan Paczyński for discussions and the OGLE group for making the light curves available over the computer network. The work is supported by a CfA postdoctoral fellowship (S.M.) and by the Science Scholars Program of the Bunting Institute (R.D.).

\section{REFERENCES}

Abt, H. A. 1983, ARA\&A, 21, 243

Alcock, C. et al. (The MACHO collaboration) 1993, Nature, 365, 621

Aubourg, E. et al. 1993b (The EROS collaboration), Nature, 365, 623

Blandford, R. D., \& Narayan, R. 1992, ARA\&A, 30, 311

Bolatto, A. D., \& Falco, E. E. 1994, Ap.J, in press

DiStefano, R. \& Mao, S. 1994, in preparation

Gould, A., \& Loeb, A. 1992, ApJ, 396, 104

Griest, K. 1991, ApJ, 366, 412

Mao, S., \& Paczyński, B. 1991, ApJ, 374, L37 
Mazeh, T., Goldberg, D., Duquennoy, A. \& Mayor, M. 1992, Ap.J, 401, 265

Paczyński, B. 1986, ApJ, 304, 1

Paczyński, B. 1991, ApJ, 371, L63

Press, W.H, Flannery, B.P., Teukolsky, S.A. \& Vetterling, W.T. 1992, Numerical Recipes (Cambridge: Cambridge Univ. Press)

Udalski, A., et al. 1993, Acta Astron. 43, 289

Udalski, A., Szymański, M., Stanek, K. Z., Kalużny, J., Kubiak, M., Mateo, M., Krzemiński, W., Paczyński, B., \& Venkat, R. 1994a, Acta Astron., 44, 165

Udalski, A., Szymański, M., Mao, S., Di Stefano, R. I., Kałużny, J., Kubiak, M., Mateo, M., Krzemiński, W. 1994b, ApJ, submitted

Udalski, A., Szymański, M., Kalużny, J., Kubiak, M., Mateo, M., Krzemiński, W. \& Paczyński, B. 1994c, Acta Astron., 44, 227

Wambsganss, J. \& Paczyński, B. 1991, AJ, 102. 864

Witt, H. J. 1990, A\&A, 236, 311 
Fig. 1.- Geometry of microlensing by a binary lens. Two lenses are located on the $\mathrm{x}$ axis with the center of mass at the origin. The separation between these two lenses is $a$. The total mass is normalized to unity and the mass ratio is given by $q=m_{2} / m_{1}$. The trajectory of the source is characterized by its impact parameter, $b$, with respect to the center of mass, the time, $t_{b}$, when the impact parameter is achieved and the angle, $\theta$, between the trajectory and the line connecting the lenses. The length scales are normalized to the Einstein radius $R_{E}$ corresponding to the total mass and the time scales are normalized to, $t_{E}$, the time needed for the source to move one Einstein radius.

Fig. 2.- A simulated light curve (circles with error bars) and the theoretical fit are shown in the left-hand panel. The "measurement" error is 0.05 mag. when the star is unlensed and becomes smaller when lensed (see text). The sampling frequency is 0.05 in arbitrary units and the total length is 8 . The source trajectory is shown in the right-hand panel as a straight line with an arrow indicating the direction of the source motion. Also shown in the right panel are the caustic structure (diamonds) and the lens positions (circles). Notice that the source trajectory intercepts the caustics for this (strong) binary event.

Fig. 3.- A simulated light curve and two binary fits are shown. The notation is the same as in Fig. 2. Notice the difference between the caustics of the two binary fits.

Fig. 4.- The light curve of the OGLE \#6 microlensing event in the I-band. Only the lensed portion is shown. The dashed and solid lines in the left-hand panel are the best point lens and the best binary fits respectively. The trajectory and caustic structure for the binary model are shown in the right-hand panel. 


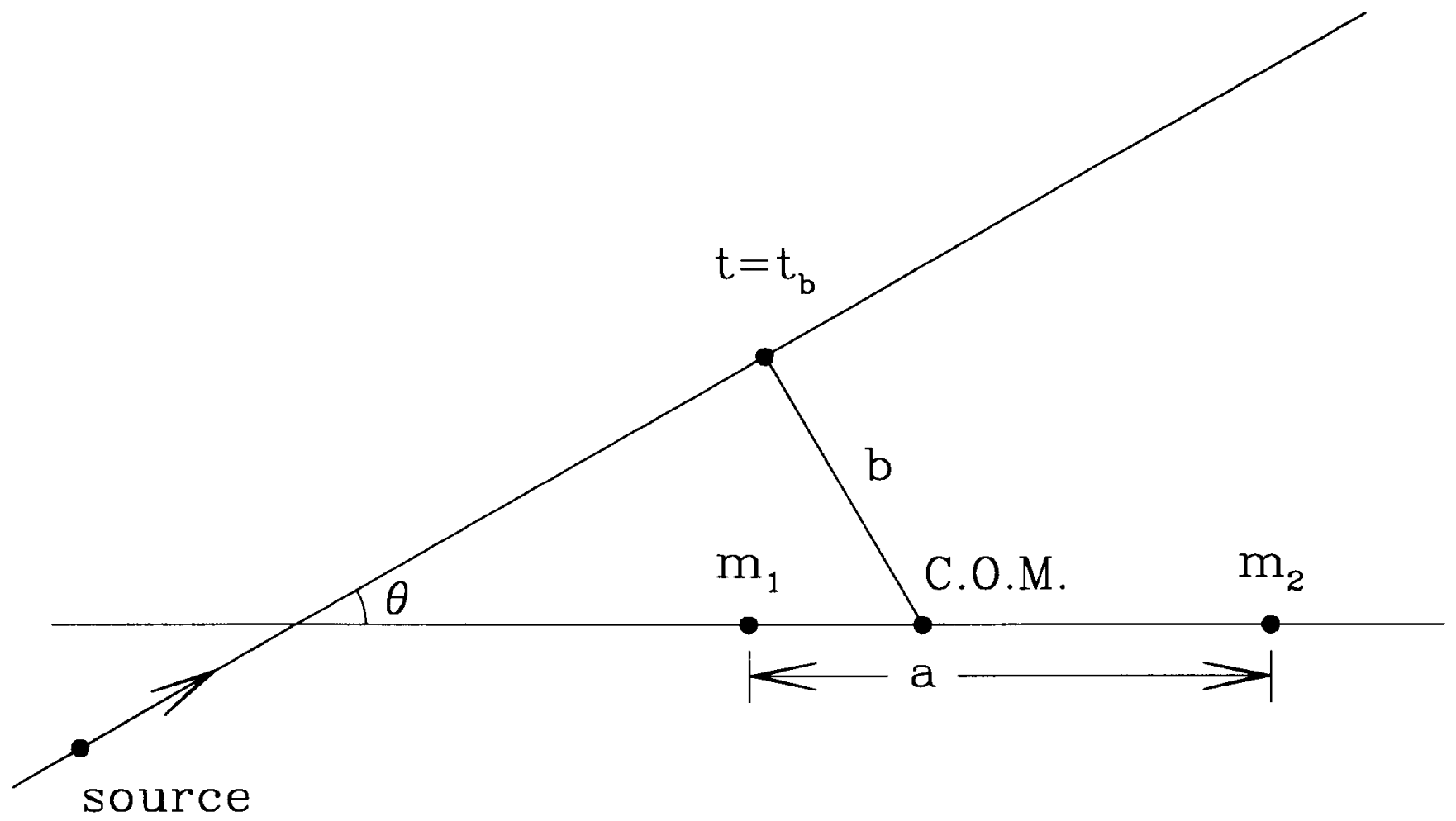

Figure 1 

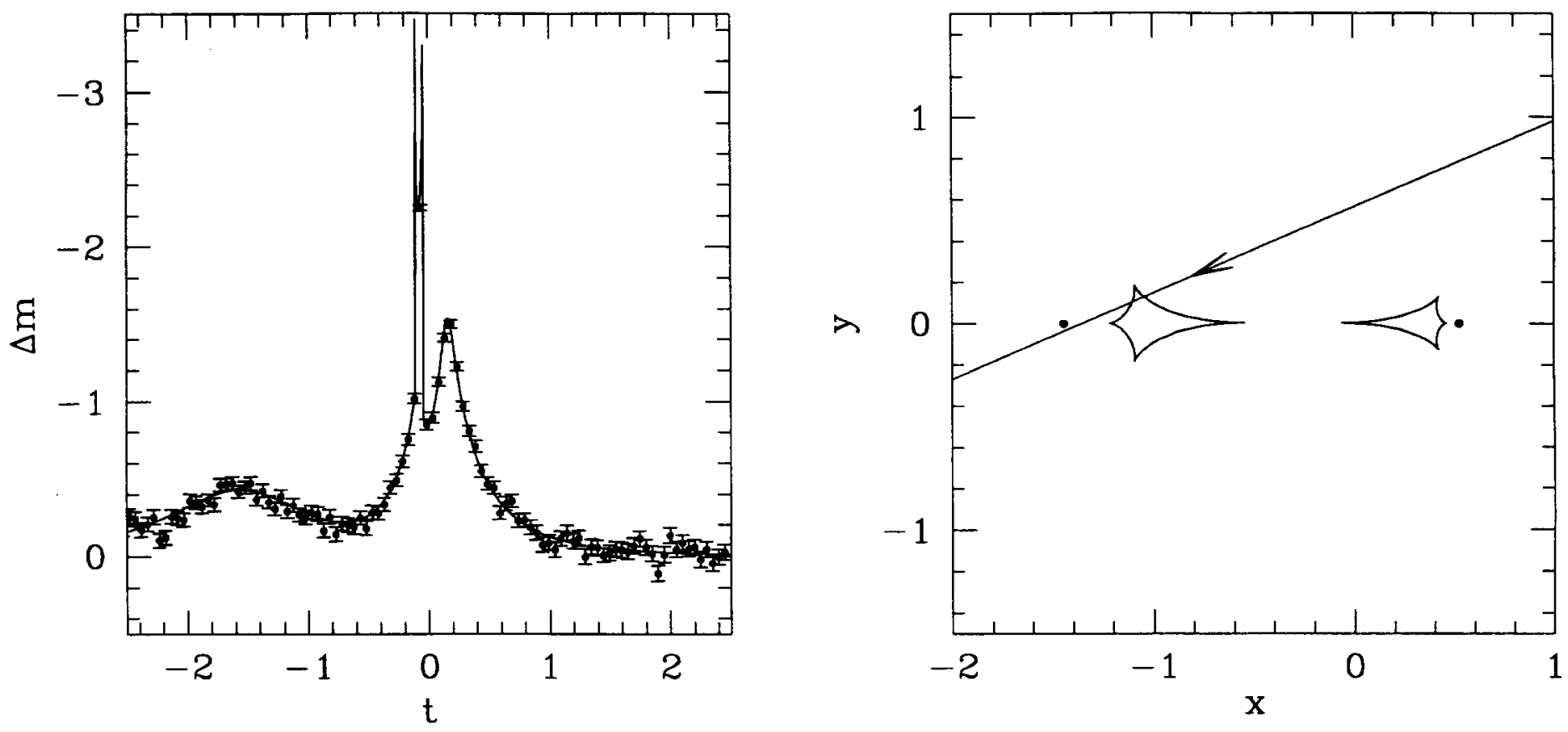

Figure 2 

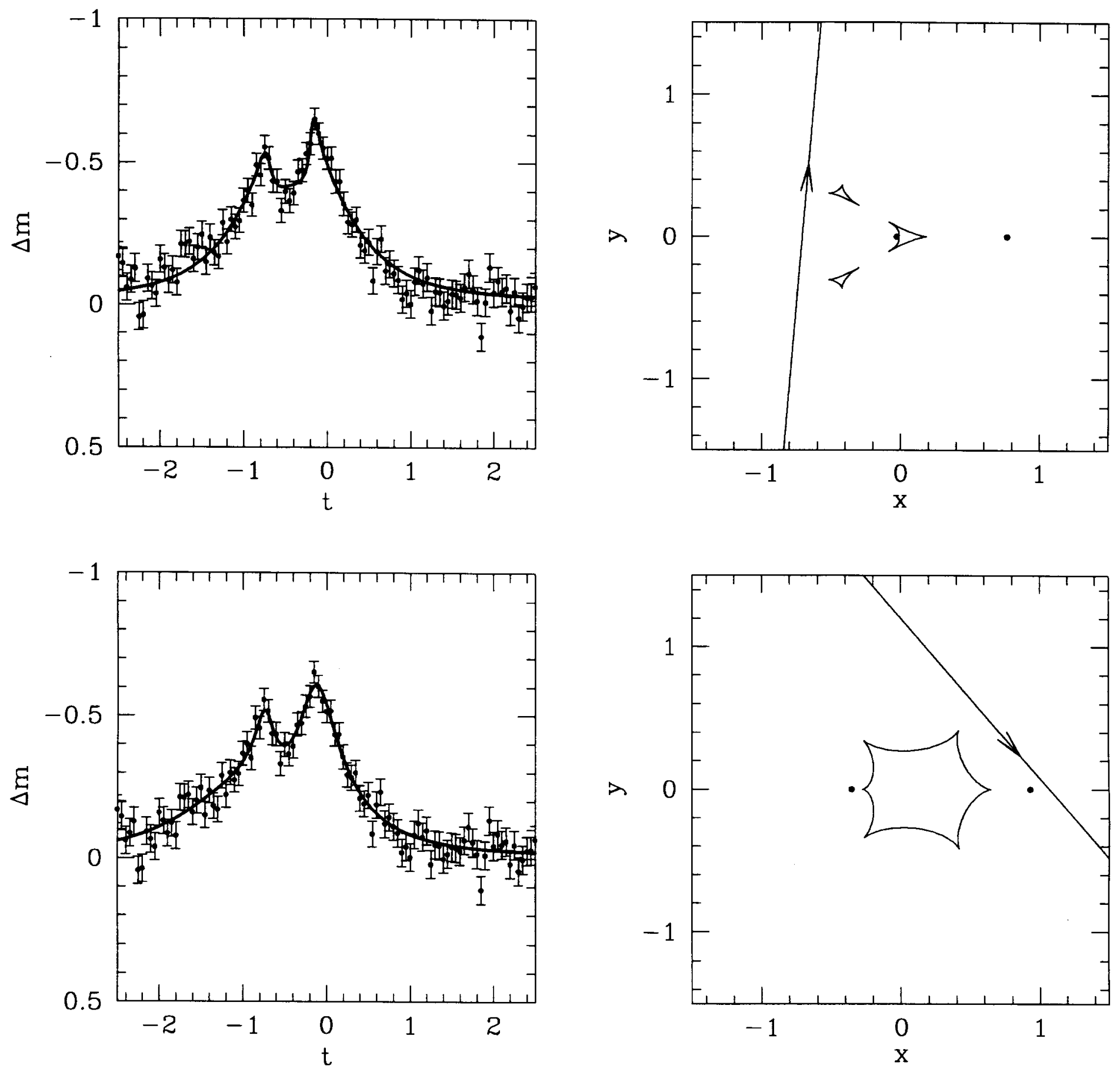

Figure 3 

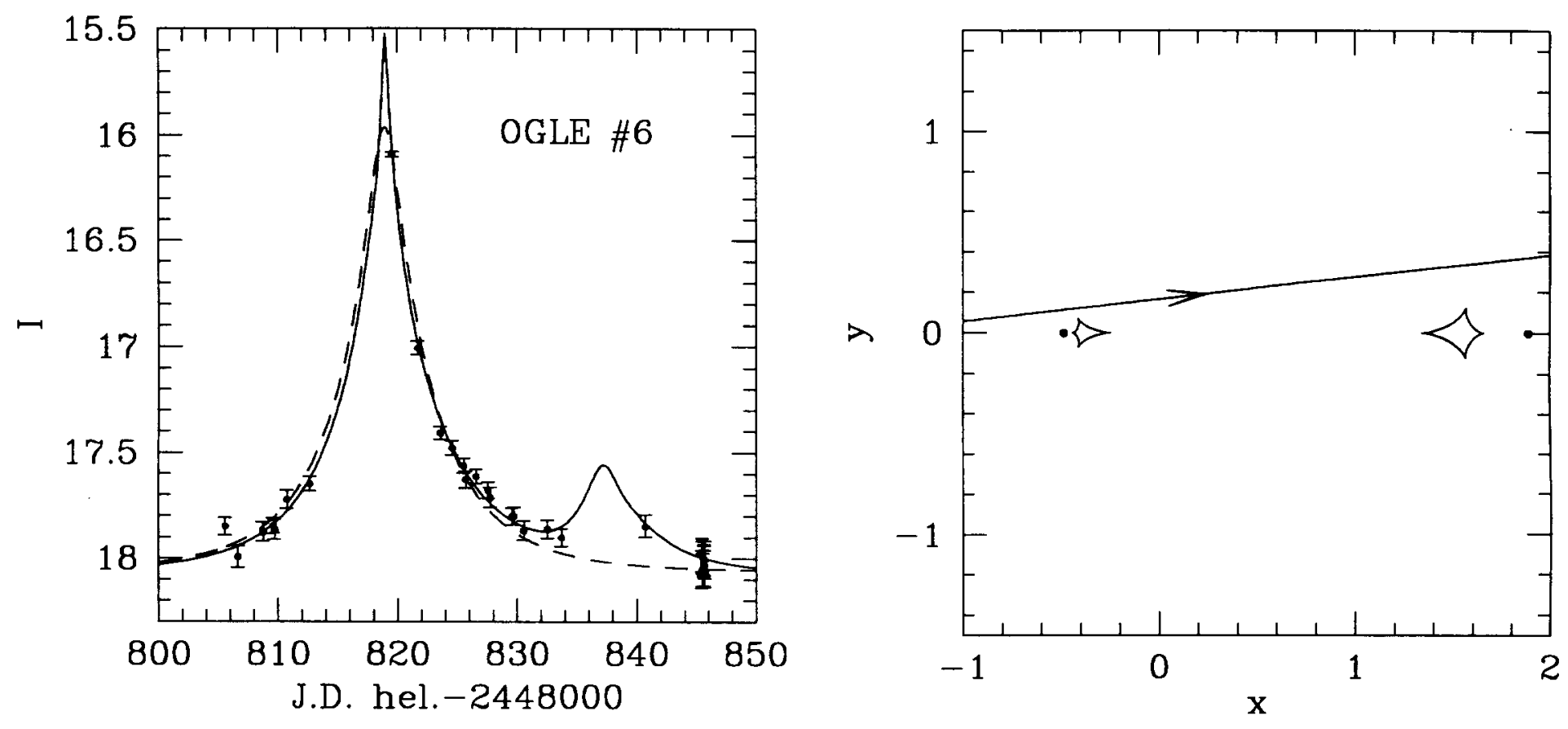

Figure 4 